\title{
Preface to special issue on 3DOR 2010
}

\author{
Ioannis Pratikakis • Tobias Schreck • \\ Theoharis Theoharis $\cdot$ Remco Veltkamp
}

Published online: 28 September 2011

(C) Springer-Verlag 2011

This special issue of The Visual Computer encompasses six papers that were selected among those presented at the 3rd Eurographics Workshop on 3D Object Retrieval (May 2, 2010, Norrköping, Sweden). The authors were invited to submit a revised and substantially extended version of their contribution to The Visual Computer journal which underwent a new review cycle.

The Eurographics Workshop on 3D Object Retrieval series, founded in 2008, provides a forum for state-of-the-art research in this exciting research field at the intersection of Computer Vision, Computer Graphics, Machine Learning, and Human-Computer Interaction.

\footnotetext{
I. Pratikakis $(\bowtie)$

Department of Electrical and Computer Engineering, Democritus University of Thrace, 67100 Xanthi, Greece

e-mail: ipratika@ee.duth.gr

url: http://utopia.duth.gr/ ipratika/
}

\section{T. Schreck}

Computer and Information Science, University of Konstanz, Universitaetsstrasse 10, Box 78, 78457 Konstanz, Germany e-mail: Tobias.Schreck@uni-konstanz.de

url: http://cms.uni-konstanz.de/informatik/schreck/

\section{T. Theoharis}

Department of Informatics and Telecommunications, University of Athens, Panepistimiopolis, 15784 Ilisia, Athens, Greece e-mail: theotheo@di.uoa.gr

url: http://graphics.di.uoa.gr

R. Veltkamp

Department of Computer Science, Utrecht University, Princetonplein 5, 3584 CC Utrecht, The Netherlands e-mail: R.C.Veltkamp@uu.nl

url: http://www.cs.uu.nl/staff/remcov.html
The 3DOR series has gathered momentum and enthusiasm from the research community addressed, and the 5th Workshop is already planned as a co-event of Eurographics 2012 in Cagliari, Italy.

3D object representations are an integral part of modern computer graphics applications, indispensible in domains such as computer-aided design, game development, biometrics, or film production. As the number of 3D models used in modern applications rapidly increases, the problem of creating new 3D models is rapidly shifting towards the problem of searching for existing 3D models in large model collections. To this end, the development of search mechanisms allowing for effective and efficient retrieval of 3D objects from large repositories is required.

The collection of six papers appearing in this special issue comprise timely problems in the field, and provide original solutions, evaluated by state-of-the-art benchmarking approaches.

In particular, Raif Rustamov presents a novel approach for the computation of interpolated Eigenfunctions from mesh boundaries, with applications in volumetric shape processing and retrieval. Ivan Sipiran and Benjamin Bustos develop and evaluate a novel, adaptive 3D interest point detector based on applying the Harris operator on 3D meshes. Hamid Laga addresses the 3D best view selection problem by solving a classification problem defined on view-based 3D descriptors. Marco Attene et al. devise a new algorithm for efficient 3D template identification, with applications in finding complementarities and part-in-whole matching. Simone Marini et al. study feature selection techniques; they assess the dependency between spectral features and effectiveness of retrieval and classification for a number of feature selection techniques. Finally, Stefano Berretti et al. introduce an automatic method for classification of facial ex- 
pressions based on SIFT descriptors extracted from facial interest points.

We thank all international reviewers, who dedicated their precious time using their expertise to shape the contents of this edition during a two-stage review process. We thank all authors for their timely contribution, and the effort made in addressing the reviewers' suggestions. Finally, our sincere thanks go to the Visual Computer Journal, for making pos- sible this special edition, documenting state-of-the-art research in the field of 3D object retrieval.

Ioannis Pratikakis,

Tobias Schreck,

Theoharis Theoharis,

Remco Veltkamp

(TVC Special Issue Guest Editors) 